\title{
Cognitive Radar for the Localization of RFID Transponders in Dense Multipath Environments
}

\author{
Klaus Witrisal*, Erik Leitinger*, Paul Meissner*, and Daniel Arnitz ${ }^{\dagger}$ \\ ${ }^{*}$ Graz University of Technology, Inffeldgasse 16c, A-8010 Graz, Austria, email: Witrisal@tugraz.at \\ ${ }^{\dagger}$ Duke University, Durham, NC, USA
}

\begin{abstract}
High-accuracy localization remains a much desired but elusive feature for passive radio transponders as used in radio-frequency identification (RFID). We believe that the principle of cognitive radar can overcome the fundamental physical limitations hindering its implementation. We propose to jointly employ a narrowband radio to interrogate the transponders and an adaptive (ultra) wideband backscatter radio for the target tracking and for actuating, sensing, and learning the radio environment. This paper explores system model and key processing steps of such a cognitive secondary radar. At its core is a perception-action cycle, which consists of transmitter and receiver-side environment models for representing radio channel conditions and Bayesian trackers for the target states. Multipath is exploited to improve the robustness and to make optimum use of the radar's sensing capabilities. Feedback information is derived from the Cramér-Rao lower bound on the position error. Initial results are presented as a basic proof of principle.

Index Terms-Ultra-wideband, Cramér-Rao lower bound, localization, multipath-assisted indoor positioning, channel modeling
\end{abstract}

\section{INTRODUCTION}

Recent seminal work by S. Haykin and co-workers described Cognitive Radar (CR) as a "step toward bridging the gap between neuroscience and engineering" [1]. The information processing in the visual brain, which has been extensively studied in neuroscience, gives valuable insight in the unparalleled capability of the brain of filtering out clutter and focusing attention on activity. In their earlier paper [2], a bat has been discussed as an example showing the efficiency of cognitive processing in nature, in a setting that closely resembles a multistatic radar. The bat adapts its emitted ultrasound pulses, e.g. while approaching its bait. In doing so, it acts on the environment in an adaptive fashion, its sonar comprises a closed perception-action cycle (PAC). Memory and attention are considered to be key facilitators of "intelligence", both of which can be mapped to signal processing functionalities of a CR [1]-[3].

A cognitive tracking and identification system is described in this paper that allows high-definition localization of large numbers of passive radio transponders ${ }^{1}$ (enhanced RFID tags) in indoor environments at decimeter accuracy. We assume that most tagged objects are static over significant time spans. The radar then learns an accurate model of the scenario including radio channel properties, based on which it assigns bandwidth

This work was partly supported by the Austrian Science Fund (FWF) within the National Research Network SISE project S10610.

1 "Passive" means that no battery is available for powering the transponder. and processing resources for real-time tracking of objects that are being moved. The proposed system aims at (pseudo) realtime tracking of up to hundreds objects per cubic meter as seen in warehouse or sales floor applications.

In this paper, we focus on the PAC having a central role in a CR. Dense multipath propagation is the dominating characteristics of the propagation environment that has to be taken into account [4]. Adaptive illumination of the environment can exploit known deterministic multipath components (MPCs) through a TX-side environment model that is learned at runtime. Time reversal (TR) processing [5], [6] is proposed, which is expected to be beneficial to overcome the degenerate pinhole channel of a backscatter radio system [7]. At the receiver side, deterministic MPCs can aid the target localization problem and overcome non-line-of-sight (NLOS) situations [8]. An RX-side environment model that is also learned at runtime provides the required channel information. This rigorous way of exploiting multipath is the key difference to the related work found in [9].

The paper is organized as follows. The system model is introduced in Section II, including channel model and position error bound. In Section III, the PAC is introduced, highlighting TX adaptation, RX signal processing, and feedback. Sections IV and V elaborate on the implementation of memory and attention. Experimental results are discussed in Section VI.

\section{System Model And Position ERror Bound}

\section{A. System concept and channel model}

A network of transmitting and receiving radar nodes may be used in a final deployment of the proposed tracking system. However, for simplicity, we consider in this paper a single transmitting node only at position $\mathbf{a}_{\mathrm{TX}}$ and a single receiving node at $\mathbf{a}_{\mathrm{RX}}$. A large number of passive targets is present in the environment at locations $\left\{\mathbf{p}_{\ell}\right\}$, each of which can identify itself by modulating its radar cross section (RCS $)^{2}$.

All RF signals propagate in a multipath environment. We write the radio channel between radar node $m \in\{\mathrm{TX}, \mathrm{RX}\}$

${ }^{2}$ In [10], a hybrid UHF and UWB interrogation system has been discussed for RFID. The UHF system is used for powering up and addressing individual RFID tags. While responding by UHF backscatter signaling, the tag will modulate its UWB-RCS simultaneously and synchronously. This known signature is used to separate the returns of RFID tags from one another and from clutter. The challenges of the UHF layer of this system are beyond the scope of this paper. However, it is noted that the UHF system can benefit from (channel) information gathered by the CR. 
and target $\ell$ using a hybrid, deterministic-stochastic channel impulse response model

$$
h_{\ell, m}\left(\tau, \mathbf{p}_{\ell}\right)=\sum_{k=1}^{K_{m}\left(\mathbf{p}_{\ell}\right)} \alpha_{k, m}^{(\ell)} \delta\left(\tau-\tau_{k, m}\left(\mathbf{p}_{\ell}\right)\right)+\nu_{\ell, m}(\tau)
$$

where the sum accounts for a set of $K_{m}\left(\mathbf{p}_{\ell}\right)$ deterministic MPCs at delays $\left\{\tau_{k, m}\left(\mathbf{p}_{\ell}\right)\right\}$ and path amplitudes $\left\{\alpha_{k, m}^{(\ell)}\right\}$, while $\nu_{\ell, m}(\tau)$ represents diffuse multipath.

We assume a deterministic model for the delays related to the geometry of multipath components. For example, reflections (of arbitrary order) at plane surfaces can be attributed to virtual transceivers at fixed locations $\mathbf{a}_{k, m}$ that result from reflecting the physical transceiver in those planes. This yields $\tau_{k, m}\left(\mathbf{p}_{\ell}\right)=\frac{1}{c}\left\|\mathbf{a}_{k, m}-\mathbf{p}_{\ell}\right\|$, where $\mathrm{c}$ is denoted as propagation velocity.

The path amplitudes are factorized into a deterministic part $\left.\gamma_{k, m}(\mathbf{p})\right|_{\mathbf{p}=\mathbf{p}_{\ell}}$ and a random component $\beta_{k, m}^{(\ell)}$. The former accounts for the path loss, deterministic phase shift, and visibility of some MPC and is equal for all targets if at location p. The latter accounts for the differences between individual transponders and for model mismatches.

The backscatter channel is composed of down and uplink channels as in (1), connected by the differential RCS $T_{\ell}(f)$ (cf. [11]) that represents the RCS difference between the binary modulation states. It will differ for individual transponders due to de-tuning, tag orientation (polarization), and production variations. We assume that these variations can be approximated by the random variables $\left\{\beta_{k, m}^{(\ell)}\right\}$ of the channel model, akin to the concept of random antenna modeling in [12]. Any errors to this model are accounted for in $\nu_{\ell, m}(\tau)$.

\section{B. Position Error Bound}

The achievable precision of multilateration systems is quantified by the position error bound (PEB), the Cramér-Rao lower bound (CRLB) of the estimated position vector $\hat{\mathbf{p}}$. We define the PEB as $\sqrt{\operatorname{tr}\left\{\mathbf{J}_{\mathbf{p}}^{-1}\right\}}$, where $\mathbf{J}_{\mathbf{p}}$ is the equivalent Fisher information matrix (EFIM) for the position vector $\mathbf{p}$ [13]. Following [8], [13], the EFIM can be given as

$$
\mathbf{J}_{\mathbf{p}}=\frac{8 \pi^{2} \beta^{2}}{c^{2}} \sum_{k=1}^{K} \operatorname{SINR}_{k} \mathbf{J}_{\mathbf{r}}\left(\phi_{k}\right)
$$

where $\beta^{2}$ is the effective (mean square) signal bandwidth,

$$
\operatorname{SINR}_{k}:=\frac{\left|\alpha_{k}\right|^{2}}{N_{0}+T_{\mathrm{s}} S_{\nu}\left(\tau_{k}\right)}
$$

is the signal-to-interference-plus-noise ratio (SINR) of the $k$-th deterministic signal component arriving at delay $\tau_{k}$ and angle $\phi_{k}$, and $\mathbf{J}_{\mathbf{r}}\left(\phi_{k}\right)$ is the so-called ranging direction matrix (cf. [13]), a rank-one matrix with an eigenvector in direction of $\phi_{k}$. "Interference" has been modeled as a Gaussian random process representing diffuse multipath [8]. Its effective power is $T_{\mathrm{s}} S_{\nu}\left(\tau_{k}\right)$, the product of an effective pulse duration $T_{s}$ and the power delay profile $S_{\nu}\left(\tau_{k}\right)$ for the diffuse multipath at delay $\tau_{k}$. This result holds for a scenario, where an active source is being localized. For backscatter channels, the PEB has a similar form, but the transceiver configuration now resembles a MIMO radar as in [14]. Furthermore, the model of the diffuse multipath has to account for the convolution of the diffuse and deterministic parts of the down and uplink channels. Initial result for this backscatter-channel PEB are presented in Section VI-B.

Equation (2) illustrates that each deterministic signal component contributes information to the localization problem ${ }^{3}$, given that its source's geometry is known. This knowledge will be provided by the RX-side environment model of the CR. Equation (2) also indicates-through the $\mathrm{SINR}_{k}$ - how much information each signal component adds, which is needed for proper weighting of measurements. These weights can also be learned by the CR. Firm insight is thus provided how to employ the CR for efficiently and robustly estimating the target locations. Evaluating (2), it has been found in [8], [15] that bandwidths of at least $1 \mathrm{GHz}$ are needed to achieve satisfying positioning performance in dense indoor channels.

\section{Perception-Action CyCle}

The PAC at the core of the CR consists of three main building blocks (cf. [1]), the Bayesian tracking filter for representing the target states, the system model library for receiver optimization, and the transmit waveform library for transmitter control. However, in contrast to [1], we propose to employ multiple Bayesian state-space trackers for representing environment and target states. The TX-side and RX-side environment models represent global information that is common to all targets, while the target state-space models represent individual information including the target positions.

\section{A. Transmitter adaptation}

Time-reversal transmitter beam forming is a well-known strategy for coping with clutter in MIMO radar systems (see e.g. [16]) but it has also been discussed for UWB (e.g. [5], [6]). Some intuitive arguments can be given, why TR processing can be a good choice for this CR, while a proof of optimality is out of scope of this paper. Most importantly, focusing the transmit power onto the target optimizes the link budget. The transmit waveform for TR processing reveals the information required at the $\mathrm{TX}^{4}$. It is written as

$$
\begin{aligned}
& s_{\ell}\left(t ; \boldsymbol{\theta}_{\mathrm{TX}}\left(\hat{\mathbf{p}}_{\ell}\right), \hat{\mathbf{x}}_{\ell}\right)= \\
& \quad \sum_{k=1}^{\hat{K}_{\mathrm{TX}}}\left(\gamma_{k, \mathrm{TX}}\left(\hat{\mathbf{p}}_{\ell}\right) \hat{\beta}_{k, \mathrm{TX}}^{(\ell)}\right)^{*} p\left(t+\tau_{k, \mathrm{TX}}\left(\hat{\mathbf{p}}_{\ell}\right)\right)
\end{aligned}
$$

where $\boldsymbol{\theta}_{\mathrm{TX}}\left(\hat{\mathbf{p}}_{\ell}\right)$ is the TX-side environment model that deterministically depends on the target position, while $\hat{\mathbf{x}}_{\ell}$ represents the current target state including the position estimate $\hat{\mathbf{p}}_{\ell}$. $\boldsymbol{\theta}_{\mathrm{TX}}\left(\hat{\mathbf{p}}_{\ell}\right)$ comprises the number of modeled deterministic

${ }^{3}$ References [8], [13] indicate that (2) holds only if path overlap is avoided. In other words, the deterministic components need to be orthogonal. For the time being, we assume this requirement to hold.

${ }^{4}$ The time index designating consecutive iterations of the PAC is skipped in these equations for notational simplicity. It is re-introduced in Section IV. 
MPCs $\hat{K}_{\text {TX }}$ and their parameters such as delay and amplitude. To achieve a deterministic relation to $\hat{\mathbf{p}}_{\ell}$, we propose to compute these parameters from the (estimated) positions of virtual transmitters at locations $\hat{\mathbf{a}}_{k, \mathrm{TX}}$, e.g. $\tau_{k, \mathrm{TX}}\left(\hat{\mathbf{p}}_{\ell}\right)=\frac{1}{c} \| \hat{\mathbf{a}}_{k, \mathrm{TX}}-$ $\hat{\mathbf{p}}_{\ell} \|$ and $\gamma_{k, \mathrm{TX}}\left(\hat{\mathbf{p}}_{\ell}\right)=\hat{A}_{k, \mathrm{TX}} \exp \left(j 2 \pi \tau_{k, \mathrm{TX}}\left(\hat{\mathbf{p}}_{\ell}\right) f_{c}\right)$, where $\hat{A}_{k, \mathrm{TX}}$ is an estimated MPC gain and $f_{c}$ is the carrier frequency ${ }^{5}$. A deterministic channel model can never be accurate enough to achieve perfect TR for each (target) location $\mathbf{p}_{\ell}$. To account for mismatches, we incorporate in the state vector $\hat{\mathbf{x}}_{\ell}$ the previously introduced gain correction factors $\hat{\beta}_{k, \mathrm{TX}}^{(\ell)}$ for each MPC. Finally, $p\left(t+\tau_{k, \mathrm{TX}}\left(\hat{\mathbf{p}}_{\ell}\right)\right)$ in (4) are transmit pulses shifted to a-causal TX times (for simplicity of notation).

The transmitter-side environment model $\boldsymbol{\theta}_{\mathrm{TX}}(\mathbf{p})$ will be learned considering the joint feedback from the receiver for an ensemble of targets, as explained in Section IV.

\section{B. Measurement Model}

The received signal, when interrogating tag $\ell$, is obtained from convolution of (4) by the up and down-link channels (1) for $m=\mathrm{TX}, \mathrm{RX}$ and the addition of WGN $w(t)$,

$$
\begin{aligned}
z_{\ell}(t)= & s_{\ell}\left(t ; \boldsymbol{\theta}_{\mathrm{TX}}\left(\hat{\mathbf{p}}_{\ell}\right), \hat{\mathbf{x}}_{\ell}\right) * h_{\ell, \mathrm{TX}}\left(t, \mathbf{p}_{\ell}\right) * h_{\ell, \mathrm{RX}}\left(t, \mathbf{p}_{\ell}\right)+w(t) \\
= & {\left[\sum_{k=1}^{\hat{K}_{\mathrm{TX}}} \gamma_{k, \mathrm{TX}}^{*}\left(\hat{\mathbf{p}}_{\ell}\right) \gamma_{k, \mathrm{TX}}\left(\mathbf{p}_{\ell}\right)\left(\hat{\beta}_{k, \mathrm{TX}}^{(\ell)}\right)^{*} \beta_{k, \mathrm{TX}}^{(\ell)}\right.} \\
& \times p\left(t+\frac{1}{c}\left\|\hat{\mathbf{a}}_{k, \mathrm{TX}}-\hat{\mathbf{p}}_{\ell}\right\|-\frac{1}{c}\left\|\mathbf{a}_{k, \mathrm{TX}}-\mathbf{p}_{\ell}\right\|\right) \\
& +\sum_{k=1}^{\hat{K}_{\mathrm{TX}}} \sum_{k^{\prime}=1, k^{\prime} \neq k}^{K_{\mathrm{TX}}\left(\mathbf{p}_{\ell}\right)} \gamma_{k, \mathrm{TX}}^{*}\left(\hat{\mathbf{p}}_{\ell}\right) \gamma_{k^{\prime}, \mathrm{TX}}\left(\mathbf{p}_{\ell}\right)\left(\hat{\beta}_{k, \mathrm{TX}}^{(\ell)}\right)^{*} \beta_{k^{\prime}, \mathrm{TX}}^{(\ell)} \\
& \times p\left(t+\frac{1}{c}\left\|\hat{\mathbf{a}}_{k, \mathrm{TX}}-\hat{\mathbf{p}}_{\ell}\right\|-\frac{1}{c}\left\|\mathbf{a}_{k^{\prime}, \mathrm{TX}}-\mathbf{p}_{\ell}\right\|\right) \\
& \left.+s_{\ell}\left(t ; \boldsymbol{\theta}_{\mathrm{TX}}\left(\hat{\mathbf{p}}_{\ell}\right), \hat{\mathbf{x}}_{\ell}\right) * \nu_{\ell, \mathrm{TX}}(t)\right] * h_{\ell, \mathrm{RX}}\left(t, \mathbf{p}_{\ell}\right)+w(t) \\
\approx & {\left[\sum_{k=1}^{\hat{K}_{\mathrm{TX}}}\left|\gamma_{k, \mathrm{TX}}\left(\mathbf{p}_{\ell}\right) \beta_{k, \mathrm{TX}}^{(\ell)}\right|^{2}\right] p(t) * h_{\ell, \mathrm{RX}}\left(t, \mathbf{p}_{\ell}\right) } \\
& +w\left(t ; \boldsymbol{\theta}_{\mathrm{TX}}\left(\hat{\mathbf{p}}_{\ell}\right), \hat{\mathbf{x}}_{\ell}\right) .
\end{aligned}
$$

The final approximation (5) assumes accurate environment models and target state estimates, i.e. we have $\hat{\mathbf{p}}_{\ell} \approx \mathbf{p}_{\ell}$ and $\hat{\mathbf{a}}_{k, \mathrm{TX}} \approx \mathbf{a}_{k, \mathrm{TX}}$ and $\gamma_{k, \mathrm{TX}}\left(\hat{\mathbf{p}}_{\ell}\right) \hat{\beta}_{k, \mathrm{TX}}^{(\ell)} \approx \gamma_{k, \mathrm{TX}}\left(\mathbf{p}_{\ell}\right) \beta_{k, \mathrm{TX}}^{(\ell)}$, $\forall k$, such that the deterministic MPCs add up coherently at the target. It comprises an additive noise component $w\left(t ; \boldsymbol{\theta}_{\mathrm{TX}}\left(\hat{\mathbf{p}}_{\ell}\right), \hat{\mathbf{x}}_{\ell}\right)$ which depends on the TX waveform parameters $\boldsymbol{\theta}_{\mathrm{TX}}\left(\hat{\mathbf{p}}_{\ell}\right)$ and $\hat{\mathbf{x}}_{\ell}$. By this dependence, the transmitter controls the accuracy of the state estimation in the RX, an essential property of a CR [1]. ${ }^{6}$

\section{Receiver processing}

Assuming the approximation (5) to hold, we can exploit the insight obtained in Section II-B to design the receiver-side signal processing. The measurement log-likelihood function is

\footnotetext{
${ }^{5}$ Note that a path-loss model is not included in this example.

${ }^{6}$ It is noted that the noise component $w\left(t ; \boldsymbol{\theta}_{\mathrm{TX}}\left(\hat{\mathbf{p}}_{\ell}\right), \hat{\mathbf{x}}_{\ell}\right)$ also represents the cross-terms $k \neq k^{\prime}$ from the intermediate convolution result.
}

approximated as the inner product of the received signal and a hypothesized channel response (cf. [8, (14)]),

$$
\begin{aligned}
\ln \mathrm{p}\left(z_{\ell}(t) \mid \boldsymbol{\psi}_{\mathrm{RX}}(\mathbf{p}), \hat{\mathbf{x}}_{\ell}\right) & \\
\approx & \frac{2}{N_{0}}\left\langle z_{\ell}(t), \sum_{k=1}^{\hat{K}_{\mathrm{RX}}} \hat{w}_{k}^{2}(\mathbf{p}) \hat{\alpha}_{k, \mathrm{RX}}^{(\ell)} p\left(t-\hat{\tau}_{k, \mathrm{RX}}(\mathbf{p})\right)\right\rangle \\
& -\frac{E_{p}}{N_{0}} \sum_{k=1}^{\hat{K}_{\mathrm{RX}}} \hat{w}_{k}^{2}(\mathbf{p})\left|\hat{\alpha}_{k, \mathrm{RX}}^{(\ell)}\right|^{2}
\end{aligned}
$$

where $E_{p}$ is the energy of pulse $p(t)$. The hypothesis is parametrized by the vector $\psi_{\mathrm{RX}}(\mathbf{p})$, whose entries comprise the RX-side environment model. In particular, we again relate the path delays to virtual receivers at fixed positions, obtaining $\hat{\tau}_{k, \mathrm{RX}}(\mathbf{p})=\frac{1}{c}\left\|\hat{\mathbf{a}}_{k, \mathrm{RX}}-\mathbf{p}\right\|$. These virtual receiver locations will be estimated by analyzing the combined measurements of an ensemble of targets, see Section IV. The environment model also provides the weights $\hat{w}_{k}^{2}(\mathbf{p})$, which quantify the power of diffuse multipath interfering with the $k$-th deterministic component (cf. Section II-B and [8]). The path gains $\left\{\hat{\alpha}_{k, \mathrm{RX}}^{(\ell)}\right\}$ are nuisance parameters to the location estimation. ${ }^{7}$

In case the environment models and/or the target state estimates are not exact, the following extended likelihood function can be employed

$$
\begin{aligned}
& \ln \mathrm{p}\left(z_{\ell}(t) \mid \boldsymbol{\psi}_{\mathrm{RX}}(\mathbf{p}), \boldsymbol{\theta}_{\mathrm{TX}}(\mathbf{p}), \hat{\mathbf{x}}_{\ell}\right) \\
& \approx \\
& \frac{2}{N_{0}}\left\langle z_{\ell}(t), \sum_{k=1}^{\hat{K}_{\mathrm{TX}}} \sum_{i=1}^{\hat{K}_{\mathrm{RX}}} \hat{w}_{i}^{2}(\mathbf{p}) \hat{\alpha}_{k, \mathrm{TX}}^{(\ell)} \hat{\alpha}_{i, \mathrm{RX}}^{(\ell)}\right. \\
& \left.s_{\ell}\left(t-\hat{\tau}_{k, \mathrm{RX}}(\mathbf{p})-\hat{\tau}_{i, \mathrm{TX}}(\mathbf{p}) ; \boldsymbol{\theta}_{\mathrm{TX}}\left(\hat{\mathbf{p}}_{\ell}\right), \hat{\mathbf{x}}_{\ell}\right)\right\rangle \\
& -\frac{1}{N_{0}} \int \mid \sum_{k=1}^{\hat{K}_{\mathrm{TX}}} \sum_{i=1}^{\hat{K}_{\mathrm{RX}}} \hat{w}_{i}(\mathbf{p}) \hat{\alpha}_{k, \mathrm{TX}}^{(\ell)} \hat{\alpha}_{i, \mathrm{RX}}^{(\ell)} \\
& \left.s_{\ell}\left(t-\hat{\tau}_{k, \mathrm{RX}}(\mathbf{p})-\hat{\tau}_{i, \mathrm{TX}}(\mathbf{p}) ; \boldsymbol{\theta}_{\mathrm{TX}}\left(\hat{\mathbf{p}}_{\ell}\right), \hat{\mathbf{x}}_{\ell}\right)\right|^{2} \mathrm{~d} t .
\end{aligned}
$$

Hence, a mismatch between the real and estimated target position $\hat{\mathbf{p}}_{\ell}$, which results in choosing the wrong TR processing parameter $\boldsymbol{\theta}_{\mathrm{TX}}\left(\hat{\mathbf{p}}_{\ell}\right)$ for the transmitter adaptation, can be accounted for. Otherwise, the likelihood function would have a systematic error, which means that its maximum were not on the target's real position.

\section{Feedback information}

The (posterior) CRLB is a suitable measure for feedback information within the PAC [1]. Suitable approximations of this quantity are subject of ongoing research. However, the

\footnotetext{
${ }^{7}$ In noncoherent processing, the path gains are estimated individually from the measurements. Coherent processing assumes knowledge of the complexvalued path gains. It has been shown in [14] that in this case the target localization error will depend on the carrier frequency of the radio signal rather than the bandwidth. This finding promises huge potential for a CR-based passive tag tracking system, because it suggests that, after an initial learning phase, the environment may be monitored very efficiently using narrowband signals instead of UWB signals. Therefore, bandwidth and carrier frequency should be incorporated in the PAC of the proposed CR, but this has been left for future work.
} 
available CRLB (2) and the measurement model (5) already indicate the type of feedback information that may be used.

- The accuracy of the TX-side TR processing determines the signal energy, noise power, and effective bandwidth of the tag reflection, cf. (5): The signal energy is proportional to the energy sum of all correctly resolved MPCs; non-resolved MPCs contribute to the noise process; incorrectly resolved pulse delays lead to a broadening of the effective transmit pulse, equivalent to decreased effective bandwidth.

- With appropriate RX-side processing, proper weighting of the MPCs is feasible. This requires the estimation of the signal-to-interference ratios of the MPCs, cf. (2).

This completes the discussion of the PAC for the proposed system. The following sections outline the roles of memory and attention.

\section{MEMORY}

We split the memory in two parts, the target states on the one hand (including the random part of the channel state information) and the environment model on the other hand. When tracking either part of the memory, we consider the other part as fixed. We proceed by describing these two estimation steps, respectively, highlighting their interdependencies.

1) State space tracking: The state of the $\ell$-th RFID tag at time step $n$ is given as $\mathbf{x}_{\ell, n}=\left[\mathbf{p} ; \mathbf{v} ; \boldsymbol{\beta}_{\mathrm{TX}} ; \boldsymbol{\beta}_{\mathrm{RX}}\right]$, where $\mathbf{p}=[x ; y]$ is the position, $\mathbf{v}=\left[v_{x} ; v_{y}\right]$ is the velocity, and $\boldsymbol{\beta}_{\mathrm{TX}}$ and $\boldsymbol{\beta}_{\mathrm{RX}}$ are the random parts of the MPC amplitudes. At this stage of our work, we consider perfect environment models and neglect the randomness of the channels of individual tags. I.e., the entries of $\left[\boldsymbol{\beta}_{\mathrm{TX}} ; \boldsymbol{\beta}_{\mathrm{RX}}\right]$ are considered to be all ones. The implementation of this random model component will be an important topic for future work.

The measurement received from the $\ell$-th tag is denoted as $\mathbf{z}_{\ell, n}$. As the states can only be observed through the measurements, we can model the tracking problem as a hidden Markov model. The posterior PDF at the $(n-1)$-th step is given by $\mathrm{p}\left(\mathbf{x}_{\ell, n-1} \mid \mathbf{z}_{\ell, 1: n-1}, \boldsymbol{\theta}_{\mathrm{TX}}, \boldsymbol{\psi}_{\mathrm{RX}}\right)$. Due to the fact that conditioned on the state at the previous time step, the current state is independent of all previous measurements, we can use the state-space transition PDF $\mathrm{p}\left(\mathbf{x}_{\ell, n} \mid \mathbf{x}_{\ell, n-1}, \mathbf{z}_{\ell, 1: n-1}\right)=$ $\mathrm{p}\left(\mathbf{x}_{\ell, n} \mid \mathbf{x}_{\ell, n-1}\right)$ to obtain the prior PDF of the target state $\mathbf{x}_{\ell, n}^{-}$ using the Chapman-Kolmogorov equation

$$
\begin{aligned}
& \mathrm{p}\left(\mathbf{x}_{\ell, n} \mid \mathbf{z}_{\ell, 1: n-1}, \boldsymbol{\theta}_{\mathrm{TX}}, \boldsymbol{\psi}_{\mathrm{RX}}\right)= \\
& \quad \int \mathrm{p}\left(\mathbf{x}_{\ell, n} \mid \mathbf{x}_{\ell, n-1}\right) \mathrm{p}\left(\mathbf{x}_{\ell, n-1} \mid \mathbf{z}_{\ell, 1: n-1}, \boldsymbol{\theta}_{\mathrm{TX}}, \boldsymbol{\psi}_{\mathrm{RX}}\right) \mathrm{d} \mathbf{x}_{\ell, n-1} .
\end{aligned}
$$

As mentioned above, the TX-side environment model $\boldsymbol{\theta}_{\mathrm{TX}}(\mathbf{p})$ is used for the TR processing. The feedback information of the PAC for choosing the optimal TR parameters can be extracted from the prior PDF (8), using the criteria discussed in Section III-D. The most simple way to obtain a specific instance of feedback information is to use the position estimate $\hat{\mathbf{p}}_{\ell, n}$ of the predicted target state $\mathbf{x}_{\ell, n}^{-}$to adapt the transmitted signal in (4), i.e.

$$
\boldsymbol{\theta}_{\mathrm{TX}}\left(\hat{\mathbf{p}}_{\ell, n}\right)=\boldsymbol{\theta}_{\mathrm{TX}}\left(\mathbb{E}\left\{\mathbf{p}_{\ell, n}^{-}\right\}\right)
$$

Hence, the new measurement $\mathbf{z}_{\ell, n}$ to be used in the update step of the state-space filter depends on the TR processing parameters $\boldsymbol{\theta}_{\mathrm{TX}}\left(\hat{\mathbf{p}}_{\ell, n}\right)$. The prior PDF (8) is updated with the measurement $\mathbf{z}_{\ell, n}$ using the Bayesian theorem, so that the posterior PDF of the updated state $\mathbf{x}_{\ell, n}^{+}$can be written as

$$
\begin{aligned}
& \mathrm{p}\left(\mathbf{x}_{\ell, n} \mid \mathbf{z}_{\ell, n}, \boldsymbol{\theta}_{\mathrm{TX}}, \boldsymbol{\psi}_{\mathrm{RX}}\right)= \\
& \frac{\mathrm{p}\left(\mathbf{z}_{\ell, n} \mid \mathbf{x}_{\ell, n}, \boldsymbol{\theta}_{\mathrm{TX}}, \boldsymbol{\psi}_{\mathrm{RX}}\right) \mathrm{p}\left(\mathbf{x}_{\ell, n} \mid \mathbf{z}_{\ell, 1: n-1}, \boldsymbol{\theta}_{\mathrm{TX}}, \boldsymbol{\psi}_{\mathrm{RX}}\right)}{\mathrm{p}\left(\mathbf{z}_{\ell, n} \mid \mathbf{z}_{\ell, 1: n-1}, \boldsymbol{\theta}_{\mathrm{TX}}, \boldsymbol{\psi}_{\mathrm{RX}}\right)}
\end{aligned}
$$

whose denominator is given by

$$
\begin{aligned}
& \mathrm{p}\left(\mathbf{z}_{\ell, n} \mid \mathbf{z}_{\ell, 1: n-1}, \boldsymbol{\theta}_{\mathrm{TX}}, \boldsymbol{\psi}_{\mathrm{RX}}\right)= \\
& \int \mathrm{p}\left(\mathbf{z}_{\ell, n} \mid \mathbf{x}_{\ell, n}, \boldsymbol{\theta}_{\mathrm{TX}}, \boldsymbol{\psi}_{\mathrm{RX}}\right) \mathrm{p}\left(\mathbf{x}_{\ell, n} \mid \mathbf{z}_{\ell, 1: n-1}, \boldsymbol{\theta}_{\mathrm{TX}}, \boldsymbol{\psi}_{\mathrm{RX}}\right) \mathrm{d} \mathbf{x}_{\ell, n} .
\end{aligned}
$$

2) Environment Memory: The second part of the memory is also implemented using a Bayesian estimator. For this step, the posterior PDFs of the state vectors $\mathbf{x}_{\ell, n}$ of the RFID tags are considered to be known. The measurements of an ensemble of RFID tags at time step $n$ are stacked into a vector $\mathbf{Z}=$ $\left[\mathbf{z}_{1, n} ; \mathbf{z}_{2, n} ; \ldots ; \mathbf{z}_{L, n}\right]$, where $L$ is the number of tags. Also, the known state parameters for time step $n$ of the ensemble of tags are stacked in a vector $\mathbf{X}=\left[\mathbf{x}_{1, n} ; \mathbf{x}_{2, n} ; \ldots ; \mathbf{x}_{L, n}\right]$. Using Bayes' theorem, one can then write the posterior PDF of the TX-side and RX-side environment models as

$$
\begin{aligned}
& \mathrm{p}\left(\boldsymbol{\theta}_{\mathrm{TX}}, \boldsymbol{\psi}_{\mathrm{RX}} \mid \mathbf{Z}, \mathbf{X}\right)= \\
& \frac{\mathrm{p}\left(\mathbf{Z} \mid \boldsymbol{\theta}_{\mathrm{TX}}, \boldsymbol{\psi}_{\mathrm{RX}}, \mathbf{X}\right) \mathrm{p}\left(\boldsymbol{\theta}_{\mathrm{TX}}, \boldsymbol{\psi}_{\mathrm{RX}} \mid \mathbf{X}\right) \mathrm{p}(\mathbf{X})}{\int \mathrm{p}\left(\mathbf{Z} \mid \boldsymbol{\theta}_{\mathrm{TX}}, \boldsymbol{\psi}_{\mathrm{RX}}, \mathbf{X}\right) \mathrm{p}\left(\boldsymbol{\theta}_{\mathrm{TX}}, \boldsymbol{\psi}_{\mathrm{RX}} \mid \mathbf{X}\right) \mathrm{p}(\mathbf{X}) \mathrm{d} \boldsymbol{\theta}_{\mathrm{TX}} \mathrm{d} \boldsymbol{\psi}_{\mathrm{RX}}} .
\end{aligned}
$$

Eq. (12) illustrates the interaction of the joint feedback and joint measurements. The former yields position-resolved instances of the TX-side environment model. These are useful for the latter, as they enable an observation of the RX-side environment model through the ensemble of tags.

Due to these two separated steps, the Bayesian state-space tracking filtering and the Bayesian estimation of the TXside and RX-side environment models can be executed in an iterative manner to get both the state of the RFID tags and the environment parameters. At this stage of our work, we focus on the first step, the tracking problem, and assume the environment as perfectly known. The modeling of the environment is part of ongoing work.

On top of these state-space models, we envision additional layers of memory that can implement large-scale models of the environment and the tag population. For instance, the changing visibility of MPCs throughout the observation area and specific characteristics of various transponders may be represented. And, when considering changing the bandwidth and carrier frequency of the TX signal, adaptations of the environment models need to be made.

\section{Attention}

We assume availability of a static propagation environment over extended periods of time. In such phases, the CR can repeatedly probe the environment and thereby incrementally learn the environment models, interrogating the targets in a round-robin fashion until convergence. 


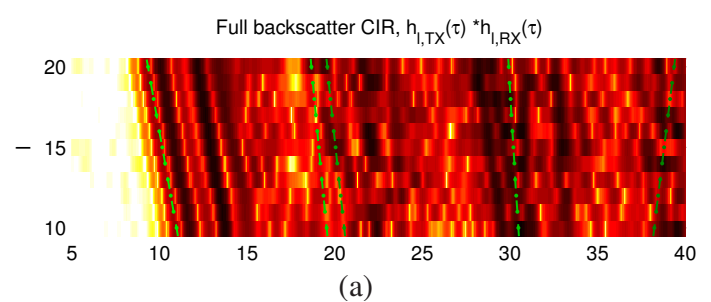

(a)

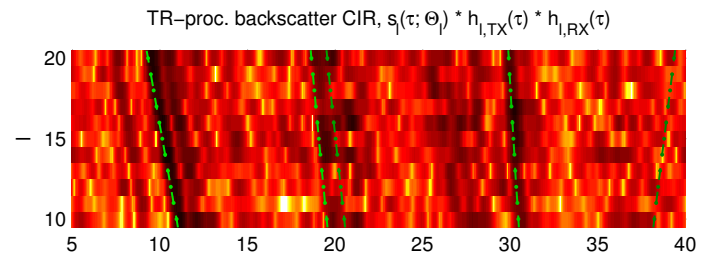

(b)

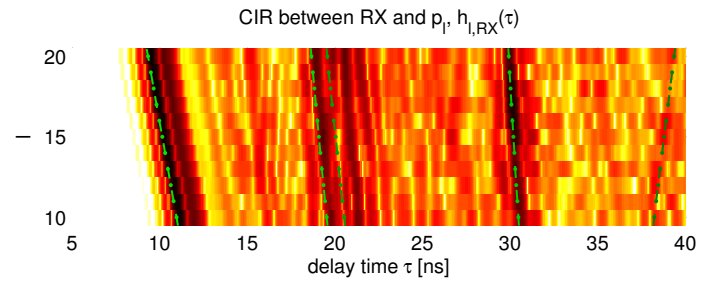

(c)

Fig. 1. Illustration of TR processing over an NLOS uplink channel. Deterministic components of the (LOS) downlink channel are indicated. (a) CIRs for the backscatter channel, the concatenation of up and downlink channels; (b) RX signals with TR processing; (c) CIRs for the downlink.

The key role of attention is providing fast and accurate response to changes within the environment. Ideally, the $\mathrm{CR}$ will respond by assigning additional resources for tracking those moving targets. Here, an adaptive signal bandwidth can be exploited again. Less bandwidth implies less accuracy but also a lower resolution of the search space. The latter will benefit the convergence of the PAC when re-localizing targets that moved "suddenly". Remember that we propose to monitor thousands of targets, hence a continuous high-rate monitoring of all targets is not feasible. Thus it is very likely that the state-space trackers have to respond to "large" movements.

\section{INITIAL RESULTS}

\section{A. Time-reversal Processing - Experimental Results}

The performance of TR processing is demonstrated in Fig. 1, based on measurement data. The up and downlink channels have been measured separately (but simultaneously) using a vector network analyzer in the $6-8 \mathrm{GHz}$ frequency range, connected to three antennas for TX, RX, and transponder. The transponder antenna was moved along a straight line in $5 \mathrm{~cm}$ steps. For this illustration, the TX and RX-side environment models have been extracted from the geometry of the environment as discussed in [15]. An NLOS situation was chosen for the uplink to demonstrate the full potential of TR. The downlink was LOS.

Fig. 1(a) illustrates the impulse response of the "full" backscatter channel, the concatenation of the up and downlink channels, without TR processing. A series of 11 measurements is depicted, allowing the eye to distinguish deterministic components from diffuse multipath. The expected MPCs of the downlink channel-corresponding to the RX-side environment model-are superimposed as dashed lines with bullet markers. It is clearly seen that this model does not match the measurement data. Any localization algorithm employing this information would fail.

Fig. 1(b) includes the TR signal according to (4). Now a good match is seen between the measurement and the expected MPCs, in particular at 10 and $30 \mathrm{~ns}$ but also at 20 ns. For comparison, the pure downlink channel is shown in Fig. 1(c). The latter reveils the same components. It shows less pronounced diffuse multipath but the information bearing timing is equivalent. Another difference is the appearance of artifacts from the TR processing in Fig. 1(b), such as spurious components arriving prior to the LOS.

\section{B. Target Tracking, Time-reversal, and PAC}

We next illustrate the impact of TR processing for waveform adaptation in the PAC on the tracking performance. The TXside and RX-side environment models are assumed to be perfectly known at this stage. A computer simulation model has been implemented, consisting of one TX node and two RX nodes to track one mobile target (MT) along a trajectory as shown in Fig. 2(a). The trajectory consists of 35 target positions, which represent 35 time steps. For the motion model we used a linear and constant velocity state-space propagation model with Gaussian process noise.

The multipath channels from the transmitter to the RFID tag and the channel from the tag to the receiver are modeled by (1). The deterministic part of the channels comprises the LOS path and first and second-order specular reflections. The transmitted pulse $\mathrm{p}(t)$ is modeled as root-raised cosine pulse, with a rolloff factor of $\beta_{\text {roll }}=0.6$ and a pulse duration of $T_{p}=0.5 \mathrm{~ns}$. The carrier frequency $f_{c}$ of the pulse is $7 \mathrm{GHz}$ and similar to [8], we used a free-space path-loss model for the MPC gains. The diffuse part of one single channel is modeled as doubleexponential function cf. (eq. (9) in [17]). Its parameters are the total power of the diffuse multipath $\Omega_{1}=2.32 \times 10^{-7}$, $\gamma_{1}=20 \mathrm{~ns}, \gamma_{\text {rise }}=5 \mathrm{~ns}$ and $\chi=0.98$. The effective SNR of the LOS components are $30 \mathrm{~dB}$ (at the first trajectory point).

A particle based method was used for the Bayesian statespace filter. We used an auxiliary sampling importance resampling particle filter as described in [18]. The PDFs are represented by 100 particles. The particles are perfectly initialized with the first trajectory point of the MT. Fig. 2(a) illustrates the evolution of the particles along the trajectory. The CDFs of the obtained position estimation errors are shown in Fig. 2(b), averaged over 60 runs.

To better understand the impact of TR employed for transmitter adaptation, the CRLB has been evaluated numerically for a single backscatter link of this scenario. Fig. 3 shows a clear advantage for the TR method. Without TR, path overlap occurs to a vast extent, due to the very large number of deterministic components in the backscatter channel (approx. 120). Path overlap leads to information loss for the overlapping 


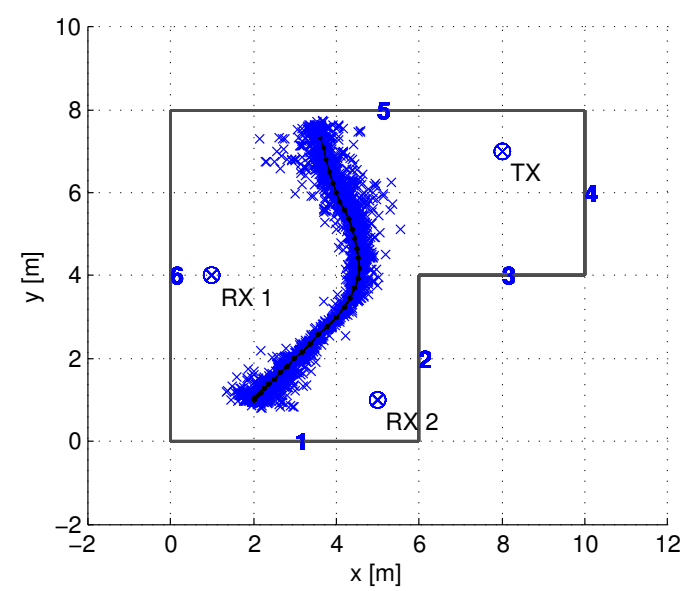

(a)

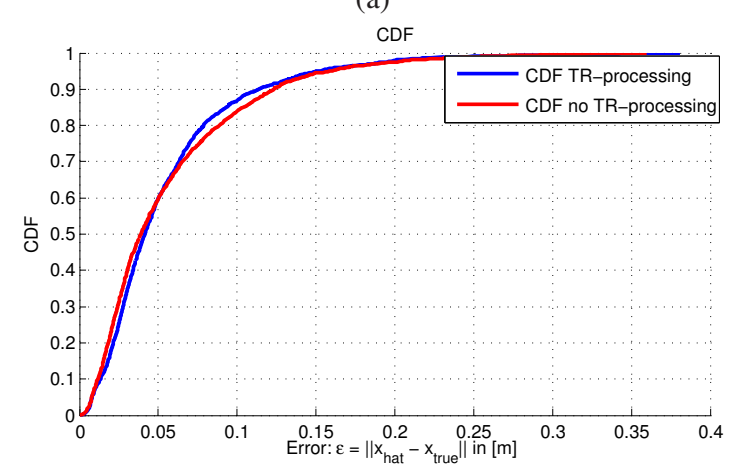

(b)

Fig. 2. (a) Simulation scenario and particles representing the target state along the trajectory. (b) Tracking performance.

deterministic components, which is evident in Fig. 3 in form of peaks in the PEB. Path overlap is greatly lowered with TR, seen in a smoother PEB at significantly lower level.

Fig. 2(b) also compares two experiments, where one does and the other does not employ TR. Here surprisingly little difference is seen between the two cases. Reason is the availability of the additional RX node. It creates two stable backscatter links, both at LOS, which yields a robust configuration for location tracking, with and without TR.

\section{CONCLUSIONS}

This paper proposes cognitive radar for tracking passive backscatter transponders (RFID tags) in dense multipath environments. Deterministic multipath components are exploited to overcome robustness issues in non-LOS situations. The radar learns TX and RX-side models of the radio channel for this purpose. Time reversal processing yields a focusing of the TX power onto the target location, which helps overcoming the tight link budget. Initially, a UWB radar is needed to be able to distinguish MPCs. However, once the environment models are known, the radar may reduce its signal bandwidth, yielding reduced processing demands and improved response times.

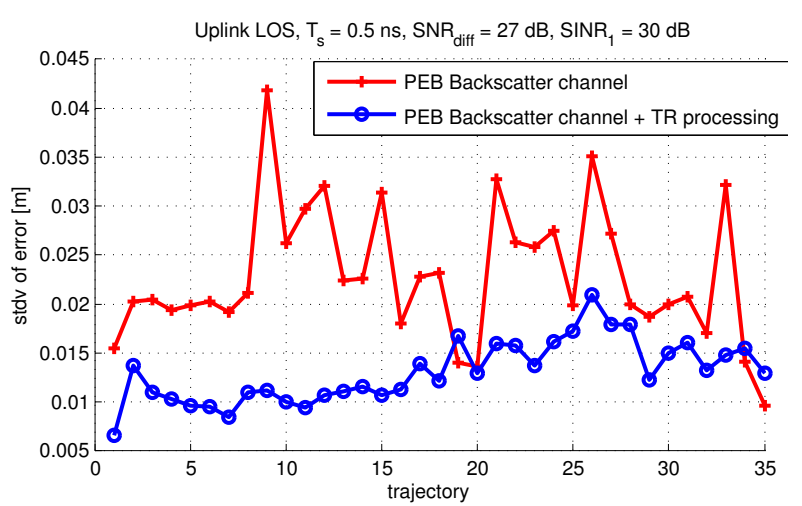

Fig. 3. CRLB of the position estimate along the trajectory shown in Fig. 2(a). The CRLB has been numerically evaluated for a single backscatter link (TX$\mathrm{RX} 1)$.

\section{REFERENCES}

[1] S. Haykin, Y. Xue, and P. Setoodeh, "Cognitive radar: Step toward bridging the gap between neuroscience and engineering," Proc. IEEE, vol. PP, no. 99, pp. $1-29,2012$.

[2] S. Haykin, "Cognitive radar: a way of the future," IEEE Signal Processing Mag., vol. 23, pp. 30-40, Jan. 2006.

[3] _ "Cognitive dynamic systems: Radar, control, and radio [point of view]," Proc. IEEE, vol. 100, no. 7, pp. 2095 -2103, Jul. 2012.

[4] A. Molisch, "Ultra-wide-band propagation channels," Proc. IEEE, vol. 97, no. 2, pp. 353-371, Feb. 2009.

[5] T. Strohmer, M. Emami, J. Hansen, G. Papanicolaou, and A. Paulraj, "Application of time-reversal with mmse equalizer to uwb communications," in IEEE Global Telecommun. Conf., GLOBECOM, vol. 5, nov.-3 dec. 2004, pp. $3123-3127$ Vol.5.

[6] N. Guo, B. Sadler, and R. Qiu, "Reduced-complexity UWB time-reversal techniques and experimental results," IEEE Trans. Wireless Commun. vol. 6, no. 12, pp. $4221-4226$, Dec. 2007.

[7] D. Arnitz, U. Muehlmann, and K. Witrisal, "Wideband characterization of backscatter channels: Derivations and theoretical background," IEEE Trans. Antennas Propagat., vol. 60, no. 1, pp. 257-266, Jan. 2012.

[8] K. Witrisal and P. Meissner, "Performance bounds for multipath-assisted indoor navigation and tracking (MINT)," in IEEE International Conference on Communications, ICC, Ottawa, Canada, Jun. 2012.

[9] Y. Chen and P. Rapajic, "Ultra-wideband cognitive interrogator network: adaptive illumination with active sensors for target localisation," vol. 4 no. 5, pp. 573-584, 262010.

[10] D. Arnitz, U. Muehlmann, and K. Witrisal, "UWB ranging in passive UHF RFID: A proof of concept," IET Electronics Letters, vol. 46, no. 20, pp. 1401-1402, Sep. 2010 .

[11] — , "Multi-frequency continuous-wave radar approach to ranging in passive UHF RFID," IEEE Trans. Microwave Theory Tech., vol. 57, no. 5, pp. 1398-1405, May 2009.

[12] A. Sibille, M. Sacko, Z. Mhanna, F. Guidi, and C. Roblin, "Joint antenna-channel statistical modelling of UWB backscattering RFID," in IEEE Intern. Conf. on Ultra-Wideband, ICUWB, Bologna, Italy, Sep. 2011.

[13] Y. Shen and M. Win, "Fundamental limits of wideband localization; Part I: A general framework," IEEE Trans. Inform. Theory, vol. 56, no. 10, pp. 4956-4980, Oct. 2010

[14] H. Godrich, A. Haimovich, and R. Blum, "Target localization accuracy gain in MIMO radar-based systems," IEEE Trans. Inform. Theory, vol. 56, no. 6, pp. 2783-2803, Jun. 2010.

[15] P. Meissner and K. Witrisal, "Analysis of Position-Related information in measured UWB indoor channels," in European Conf. on Antennas and Propagation, EuCAP, Prague, Czech Republic, Mar. 2012.

[16] J. Moura and Y. Jin, "Time reversal imaging by adaptive interference canceling," IEEE Trans. Signal Processing, vol. 56, no. 1, pp. $233-247$, Jan. 2008.

[17] J. Karedal, S. Wyne, P. Almers, F. Tufvesson, and A. F. Molisch, "A measurement-based statistical model for industrial ultra-wideband 
channels," IEEE Trans. Wireless Commun., vol. 6, no. 8, pp. 3028-3037, Aug. 2007.

[18] M. Arulampalam, S. Maskell, N. Gordon, and T. Clapp, "A tutorial on particle filters for online nonlinear/non-gaussian bayesian tracking," IEEE Trans. Signal Processing, no. 2, pp. 174 -188, feb 2002. 